\title{
BMJ Open Countermeasures against COVID-19: how to navigate medical practice through a nascent, evolving evidence base - a European multicentre mixed methods study
}

\author{
Fabian Eibensteiner (10 , ${ }^{1,2}$ Valentin Ritschl, ${ }^{3,4}$ Tanja Stamm (1) , ${ }^{3,4}$ Asil Cetin, ${ }^{5}$ \\ Claus Peter Schmitt, ${ }^{6}$ Gema Ariceta, ${ }^{7}$ Sevcan Bakkaloglu, ${ }^{8}$ \\ Augustina Jankauskiene, ${ }^{9}$ Günter Klaus, ${ }^{10}$ Fabio Paglialonga, ${ }^{11}$ Alberto Edefonti, ${ }^{11}$ \\ Bruno Ranchin, ${ }^{12}$ Rukshana Shroff, ${ }^{13}$ Constantinos J Stefanidis, ${ }^{14}$ \\ Johan Vandewalle, ${ }^{15}$ Enrico Verrina, ${ }^{16}$ Karel Vondrak, ${ }^{17}$ Aleksandra Zurowska, ${ }^{18}$ \\ Seth L Alper, ${ }^{19}$ Christoph Aufricht ${ }^{1}$
}

To cite: Eibensteiner $F$, Ritschl V, Stamm T, et al. Countermeasures against COVID-19: how to navigate medical practice through a nascent, evolving evidence base - a

European multicentre mixed methods study. BMJ Open 2021;11:e043015. doi:10.1136/ bmjopen-2020-043015

- Prepublication history and additional materials for this paper is available online. To view these files, please visit the journal online (http://dx.doi. org/10.1136/bmjopen-2020043015).

Received 22 July 2020 Revised 30 0ctober 2020 Accepted 01 February 2021

\section{Check for updates}

(C) Author(s) (or their employer(s)) 2021. Re-use permitted under CC BY-NC. No commercial re-use. See rights and permissions. Published by BMJ.

For numbered affiliations see end of article.

\section{Correspondence to}

Professor Christoph Aufricht: christoph.aufricht@meduniwien. ac.at

\section{ABSTRACT}

Objectives In a previously published Delphi exercise the European Pediatric Dialysis Working Group (EPDWG) reported widely variable counteractive responses to COVID-19 during the first week of statutory public curfews in 12 European countries with case loads of 4-680 infected patients per million. To better understand these wide variations, we assessed different factors affecting countermeasure implementation rates and applied the capability, opportunity, motivation model of behaviour to describe their determinants.

Design We undertook this international mixed methods study of increased depth and breadth to obtain more complete data and to better understand the resulting complex evidence.

Setting This study was conducted in 14 paediatric nephrology centres across 12 European countries during the COVID-19 pandemic.

Participants The 14 participants were paediatric nephrologists and EPDWG members from 12 European centres.

Main outcome measures 52 countermeasures clustered into eight response domains (access control, patient testing, personnel testing, personal protective equipment policy, patient cohorting, personnel cohorting, suspension of routine care, remote work) were categorised by implementation status, drivers (expert opinion, hospital regulations) and resource dependency. Governmental strictness and media attitude were independently assessed for each country and correlated with relevant countermeasure implementation factors.

Results Implementation rates varied widely among response domains (median $49.5 \%$, range $20 \%-71 \%$ ) and centres (median $46 \%$, range $31 \%-62 \%$ ). Case loads were insufficient to explain response rate variability. Increasing case loads resulted in shifts from expert opinion-based to hospital regulation-based decisions to implement additional countermeasures despite increased resource dependency. Higher governmental strictness and positive
Strengths and limitations of this study

- This is the first study using a mixed methods approach to evaluate and better understand the most important drivers of behaviours conducive to counteracting the COVID-19 pandemic during the first week of public curfews.

- To understand the generic mechanisms of our responses to COVID-19, the capability, opportunity, motivation model of behaviour allows for a more critical review and appraisal of current practices than standardised responses usually provided by policymakers and societal guidelines.

- Put into general context, these dynamic domains with manifold factors may provide some of the most important guiding principles but lack general completeness and might be rapidly outdated.

- Interpretation of the results of this study is limited by the small number of participating centres and mixed methods character of this study, wherefore statistical tests and their corresponding $p$ values should be interpreted with caution.

- Although our results are representative for paediatric dialysis, they may not represent to the same degree other medical responses to the COVID-19 pandemic.

media attitude towards countermeasure implementation were associated with higher implementation rates.

Conclusions COVID-19 countermeasure implementation by paediatric tertiary care centres did not reflect case loads but rather reflected heterogeneity of local rules and of perceived resources. These data highlight the need of ongoing reassessment of current practices, facilitating rapid change in 'institutional behavior' in response to emerging evidence of countermeasure efficacy. 


\section{INTRODUCTION}

SARS-CoV-2-related disease (COVID-19) spread throughout Europe when minimal evidence was available to support efficacy of then available countermeasures. ${ }^{1-4}$ The European Pediatric Dialysis Working Group (EPDWG) conducted a Delphi exercise over 5 days during the first week of statutory public curfews in 13 paediatric nephrology centres from 11 European countries ${ }^{5}$ using 'crowd intelligence' to define countermeasures in several relevant response domains, and to assess their implementation rates. ${ }^{5}$ Whereas some countermeasures (replacement of routine visits by telephone calls) were widely implemented, others (asymptomatic staff member testing) were rarely implemented, and implementation rates varied widely among countermeasures and centres. ${ }^{5}$ This heterogeneity may have reflected country-specific infection rates and pandemic stage-dependent measures to decrease infection rates. However, the mechanisms underlying COVID-19 countermeasure implementation by individual centres were not studied.

The capability, opportunity, motivation model of behaviour (COM-B $)^{6}$ describes determinants of behaviour, ${ }^{7}$ including capability (physical and psychological capacity to engage in an activity, such as knowledge and skills), opportunity (physical and social factors outside the individual that permit or prevent a certain behaviour) and motivation (brain processes that energise and direct behaviour). ${ }^{6}$ In 2011, the behaviour change wheel (BCW) was added to the COM-B to distinguish between interventions (activities aimed at changing behaviour) and policies (actions of responsible authorities or the government that enable interventions, respectively change of behaviour).$^{6}$

In this study, in order to explain the huge response variability among these tertiary care centres, we explored factors affecting practice behaviour changes for the implementation of countermeasures in each paediatric dialysis centre. We therefore used the COM-B and the BCW to map and conceptualise the determinants of behavioural change in paediatric tertiary care centres relating to COVID-19 countermeasure implementation rates during the first week of statutory public curfews in Europe. Such insights may permit improved management of impending COVID-19 resurgence(s) and of future pandemic events, especially on how to implement evidence-based changes in practice to optimise management of complex healthcare interventions.

\section{MATERIALS AND METHODS}

The methodology of the Delphi exercise conducted among the EPDWG in March 2020 was recently described. ${ }^{5}$ This follow-up study examines 14 EPDWG centres from 12 countries (Austria, Belgium, Czech Republic, France, Germany, Greece, Italy, Lithuania, Poland, Spain, Turkey and the UK). Exploration of complex and pluralistic contexts, such as cross-national studies, requires a comprehensive research approach. The mixed methods design is an ideal means to gain both depth and breadth. It allows the researcher to gain a better understanding of the research problem by yielding more complete evidence. ${ }^{8-10}$ Therefore, individual sets of 52 countermeasures (see ref 5 ) were mailed to each centre to validate countermeasure implementation rates on $20 \mathrm{March}$, and to assess altered rates on 3 April 2020. Participants were asked whether implementation decisions concerning individual countermeasures were based on expert opinion and/or hospital regulations and/or resource availability. Country-specific case loads from the European Centre for Disease Prevention and Control ${ }^{11}$ were calculated as case number per million (from Eurostat ${ }^{12}$ ). Pandemic phase was expressed as binary logarithm of case loads per million, since exponential case doubling times in the EPDWG countries ( 2 days at that time) transitioned gradually to a logistic function.

\section{Behaviour change determinants}

COM-B and BCW components were mapped to concepts derived from anonymised EPDWG experts' initial open email replies to the first Delphi exercise. ${ }^{5}$ Mapping was conducted by component definitions and experts' wording using modified meaning condensation analysis to aggregate experts' statements in terms of underlying concepts (figure 1). For example, the email statement 'Timely recipient testing should be feasible in our center' was mapped to Opportunity (physical) and to BCW policy 'environmental/social planning', whereas the statement 'I read a lot about this, but to my knowledge we cannot draw any firm conclusions' was assigned to Capability (psychological) and to BCW policy 'guidelines' (figure 1C). To ensure accuracy and rigour, initial mapping performed by one researcher (FE) was independently reviewed by a second senior qualitative researcher (VR). In cases of disagreement, consensus was achieved through discussion.

\section{Governmental strictness}

Country-specific online news agencies and governmental information websites were searched for governmental interventions in response to COVID-19. Relative frequencies of 23 defined governmental interventions to achieve 'social distancing' were combined to yield a governmental strictness score (online supplemental table 1). Interventions included restriction of free public movement, restriction of hospital access, restriction of prison access, recommended or mandatory teleworking, requirements for adequate mouth and nose coverage in public, closure of parks and playgrounds, closure of governmental facilities (eg, schools, universities), closure of mass events, recommendation to limit gatherings to five people, prohibition of gatherings exceeding five people, police surveillance, closure of non-essential businesses, closure of restaurants, local quarantine, nationwide quarantine, selective border closure, complete border closure, state of emergency, vacation ban for healthcare professionals, implementation of telemedicine, export and sales ban on all FFP3-type respirators and selected medications, ban on minors leaving home unaccompanied by a legal guardian and censorship of medical personnel. 
A)

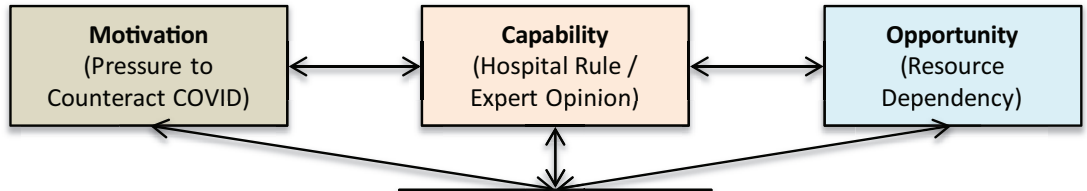

Behavioural Change

(Implementation of

Counteractive Measure)

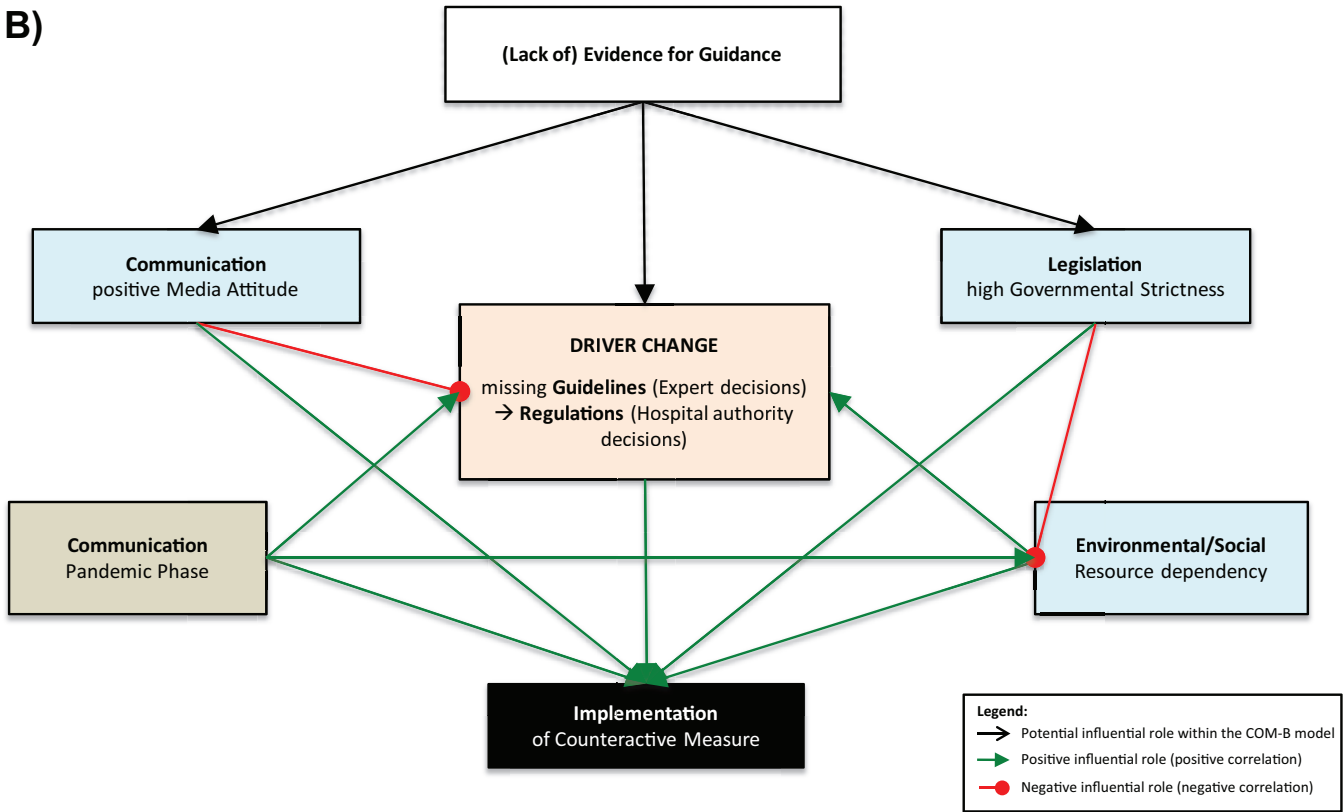

C)

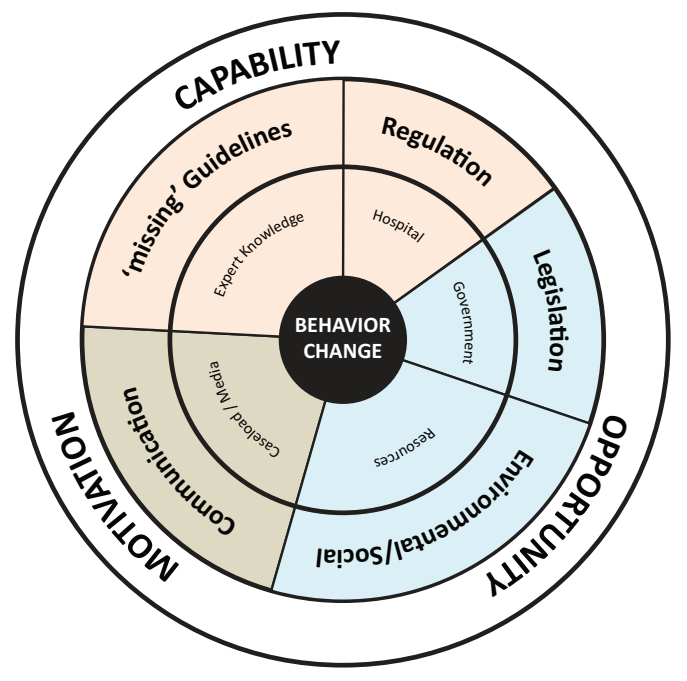

Figure 1 (A) The conceptual framework of COM-B is based on interaction between capability, opportunity and motivation to change behaviour. To implement countermeasures, expert opinion and/or hospital rules balance resource dependency of a given measure with the pressure to counteract COVID-19 during the progressive pandemic phases. (B) Factors relevant in implementing countermeasures and their interactions structured according to COM-B and the behaviour change wheel (positively correlated, green arrows; negatively correlated, red drumsticks). (C) Behaviour change wheel within the COM-B model displaying the five policy measures, with their respective concepts, influencing behaviour change as implementation of countermeasures according to the European Pediatric Dialysis Working Group (EPDWG). 


\section{Media attitude}

Cover page articles during the week of 20 March 2020 from the three widest circulating newspapers in each EPDWG country and text blocks containing COVID-19related news and/or opinion pieces were manually classified. Transcribed, translated and anonymised excerpts from the selected articles were rated by participants $(n=5)$ for positivity of reporting attitude on COVID-19 countermeasures on a scale from 1 (lowest) to 5 (highest). Excerpts were uniformly formatted without country identifiers. Mean values yielded a country-specific media attitude score (online supplemental table 1).

\section{Data analysis and statistics}

Data were clustered into eight response domains (access control, patient testing, testing healthcare personnel (HCP), personal protective equipment (PPE) policy, patient cohorting, HCP cohorting, suspension of routine care, remote work) and visualised as implementation rates and their rates of change (online supplemental figure 1). Response rates (\%) were calculated as numbers of implemented countermeasures divided by numbers of total identified countermeasures for 20 March and 3 April 2020. Resource dependency (\%) for 20 March 2020 was calculated as numbers of decisions for which resources were decisive for implementation, divided by numbers of total identified countermeasures.

Expert decisions and hospital authority decisions were expressed as the hospital authority decisions to expert decisions (H/E) ratio for 20 March 2020:

$$
\frac{H}{E} \text { Ratio }=\frac{(\text { Hospital authority decisions }(n)-\text { Expert decisions }(n))}{\text { total countermeasures }(n)}
$$

The $\mathrm{H} / \mathrm{E}$ ratio expresses the degree to which response rates are influenced by hospital authority decisions (resulting in positive values to +1 ) or by expert decisions (resulting in negative values to -1 ), with the balanced $\mathrm{H} / \mathrm{E}$ ratio of zero reflecting equivalent contributions of hospital authority and experts' decisions.

Each of these variables was calculated (1) on the domain level, as mean for each domain across all centres, and (2) on the centre level, as mean for each centre across all domains. Data were analysed with descriptive statistics using scatter plot matrices, bar plots, histograms and heat maps. Kendall's tau correlation analysis was conducted within a correlation matrix for each dependent and independent variable on each level. Correction for multiple testing was not performed, reflecting the exploratory character of this analysis. For Kendall's tau, correlation analysis between response rates and pandemic phase outliers was omitted post hoc (high response despite low case load, or relatively low responses despite highest case loads).

\section{RESULTS}

Implementation of individual counteractive measures varied widely among response domains and centres in the cross-sectional analysis of 20 March 2020. Domain response rates ranged from $20 \%(28 / 140)$ to $71 \%$ $(59 / 84)$; median $49.5 \%$. Centre response rates ranged from $31 \%(16 / 52)$ to $62 \%(32 / 52)$; median $46 \%$. Reassessment of response rates on 3 April demonstrated increased countermeasure implementation, particularly in centres with lower initial response rates ('catch-up implementation').

\section{'Snapshot' of implemented COVID-19 countermeasures (20 March 2020)}

Centre response rates or individual countermeasure response rates correlated weakly with centre case loads. Figure 2 demonstrates that centres at both ends of the pandemic phase spectrum markedly deviated from the assumption of correlation. Although overall correlations between centre responses and pandemic phase were statistically significant, country/centre-specific case loads correlated with implemented countermeasures only after outlier exclusion (table 1).

\section{Policy measures influencing implementation of countermeasures per BCW}

Five of seven BCW-defined policy measures ${ }^{6}$ were reported as reasons for behaviour change in the clinical setting (figure 1C). As expected, 'regulation' by employers (establishing rules of principles of behaviour) and/or governmental 'legislation' were important reasons for behavioural changes at the centres. However, information from mass media ('communication'), missing 'guidelines' and 'environmental/social'-related restrictions were equally often determinative for change in behavioural patterns. 'Fiscal measures' and 'service provision' were not mentioned as influencing behavioural changes. Mass media information indicated increasing pressure from growing case loads in the EPDWG centres ('communication'), corresponding to correlation of pandemic phase with average countermeasure implementation rates (table 1). Respondents often noted that recommendations ('guidelines') for clinical decision-making remained lacking, likely explaining why rules and principles established by hospital management ('regulation') contributed more as important drivers for implementation than did 'guidelines' (table 1). Growing mass media pressure ('communication') in most centres resulted in a pandemic phase-dependent shift from expert opinion (missing 'guidelines') to hospital-based 'regulations' (table 1).

Resource dependency was a major inhibitor of countermeasure implementation ('environmental/social' restrictions). Estimated resource dependency of eight individual measures correlated negatively with their implementation rates at the domain level (figure 3, table 1). Increasing resource dependency associated with an increasing ratio of hospital rules ('regulations') over expert opinion (missing 'guidelines') as a driver of countermeasure implementation (table 1). Interestingly, implementation rates for countermeasures of comparable resource 


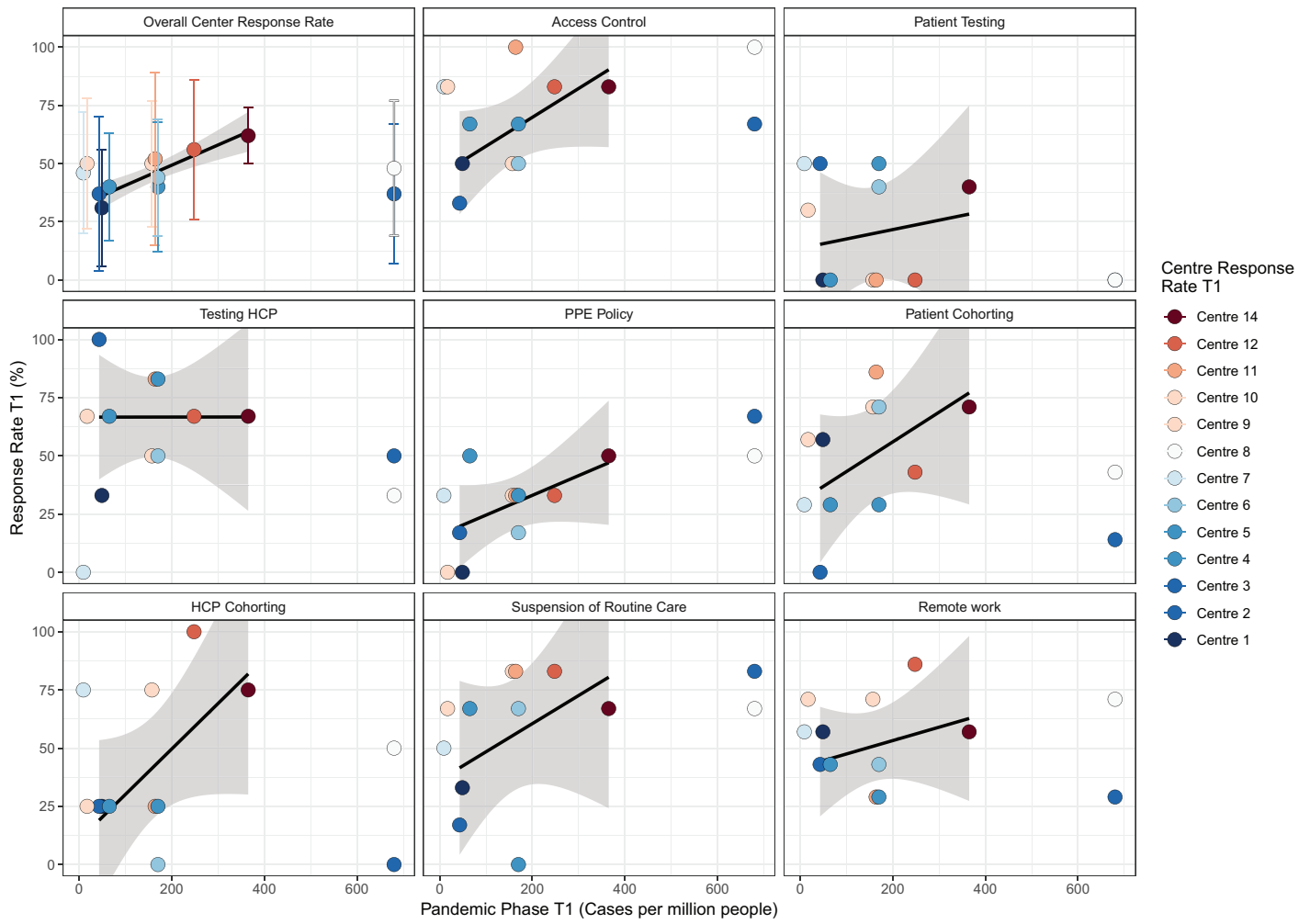

Figure 2 Response rates (calculated as relative frequencies) of implemented countermeasures for each centre ('mean center response rate', corresponding to the centre columns of online supplemental figure 1) and for each of the eight defined domains per centre, displayed as functions of pandemic phase (expressed as infected cases per million people) on 20 March 2020 (T1). Colours depicting centre response rates range from lowest (dark blue) to highest (dark red). Linear regression lines calculated after outlier exclusion (corresponding to $\left(^{*}\right)$ in table 1 ) are plotted (black) with $95 \% \mathrm{Cl}$ in grey. HCP, healthcare personnel; PPE, personal protective equipment.

dependency ('environmental/social' restrictions) increased in direct proportion to the $\mathrm{H} / \mathrm{E}$ ratio ('regulations'; compare, for example, 'Suspension of Routine Care/Remote Work' with comparably low-resource dependency and 'Testing HCP/Patients' with comparably high-resource dependency; figure 3B, figure 4).

\section{Longitudinal assessment of 'catch-up' implementation of COVID-19 countermeasures}

The above cross-sectional assessment describes associations between individual factors and countermeasure implementation rates in different centres/countries at different pandemic phases. Longitudinal changes in countermeasure implementation rates were assessed by another survey on 3 April 2020 and plotted as a function of pandemic phase. Figures $3 \mathrm{~A}$ and $4 \mathrm{~A}$ show that pandemic progression resulted in globally increased rates of countermeasure implementation from 20 March to 3 April in almost all centres (table 1). At the centre level, mean changes of response rates were negatively influenced by cumulative local perception of resource dependency on 20 March (='resource awareness', perceived 'costs'; table 1). However, 'catch-up implementation' of counteractive measures from 20 March to 3 April positively correlated with higher $\mathrm{H} / \mathrm{E}$ ratio (between hospital rule and expert opinion as drivers), and with resource dependency of particular measures (table 1). Thus, growing pressures of increased country-specific case loads increased local implementation of hospital rules, thereby overcoming the initially inhibitory effects of locally perceived resource dependency for these measures in a centre-specific way.

\section{Role of country-specific, non-medical influencers on countermeasure implementation}

Centre-specific patterns of longitudinal changes suggest that local countermeasure implementation rates represent a balance of local influences only poorly modulated by global medical evidence, allowing study of the influence of non-medical drivers such as media and government. Media attitude (online supplemental table 1) shows scores for implementation of COVID-19 countermeasures in the 11 EPDWG countries. Cover page articles from the three widest circulation newspapers during the week of 20 March each contained $>75 \%$ of COVID19-related text. Media attitude was only weakly associated with centre response rates (table 1). However, centres in countries with higher media attitude scores demonstrated significantly lower ratios of hospital rules over expert opinion (table 1), in turn associated with higher implementation rates and catch-up (table 1). Indeed, the two centres with the highest media attitude scores demonstrated the highest response rates. Online supplemental table 1 also shows governmental strictness scores of the EPDWG countries. As for media attitude, governmental strictness associated only weakly with response 
Table 1 Statistical analysis of the COM-B model as applied to the EPDWG decisions

\begin{tabular}{|llcl}
\hline COM-variable & Variable (B or COM) & Kendall's tau & P value \\
\hline Cross-sectional analysis & & & \\
\hline Pandemic phase (case load) & Implementation rate & 0.23 & $<0.01$ \\
\hline Pandemic phase (case load)* & Implementation rate* & 0.77 & $<0.01$ \\
\hline Pandemic phase (case load) & Hospital to expert ratio (centre) & 0.24 & $<0.01$ \\
\hline Hospital to expert ratio (centre) & Implementation rate & 0.41 & $<0.01$ \\
\hline Hospital to expert ratio (domain) & Implementation rate & -0.36 & $<0.01$ \\
\hline Resource dependency (centre) & Implementation rate & 0.16 & 0.03 \\
\hline Resource dependency (centre) & Hospital to expert ratio (centre) & 0.45 & $<0.01$ \\
\hline Pandemic phase (case load) & Resource dependency (centre) & 0.30 & $<0.01$ \\
\hline Resource dependency (domain) & Hospital to expert ratio (domain) & 0.47 & $<0.01$ \\
\hline Longitudinal analysis & & & 0.04 \\
\hline Implementation rate & Catch-up implementation & -0.15 & 0.01 \\
\hline Resource dependency (centre) & Catch-up implementation & -0.18 & $<0.01$ \\
\hline Resource dependency (domain) & Catch-up implementation & 0.4 & $<0.01$ \\
\hline Hospital to expert ratio (domain) & Catch-up implementation & 0.47 & \\
\hline Influence of media attitude and governmental strictness & & 0.02 \\
\hline Media attitude & Implementation rate & 0.17 & $<0.01$ \\
\hline Media attitude & Hospital to expert ratio (centre) & -0.31 & $<0.01$ \\
\hline Governmental strictness & Implementation rate & 0.3 & $<0.01$ \\
\hline Governmental strictness & Resource dependency (centre) & -0.36 & \\
\hline "Al
\end{tabular}

${ }^{*}$ After omitting outliers (=high responses despite low case load or relatively low responses despite highest case loads).

B, behaviour change; COM, capability, opportunity, motivation; EPDWG, European Pediatric Dialysis Working Group.

rates (table 1). However, centres in countries with higher governmental strictness scores demonstrated lower perceptions of resource dependency regarding countermeasure implementation (table 1), in turn associated with higher implementation rates and catch-up implementation (table 1). Interestingly, positive media attitude (potentially enhancing motivation) paired with high governmental strictness (potentially reducing resource dependency) was found in the two countries with the highest response rates (at intermediate case load).

\section{DISCUSSION}

During the COVID-19 pandemic, the most important motivational driver of behaviours conducive to counteracting the pandemic has been the magnitude of pandemic growth. In the absence of prior evidence, many interventions were rapidly executed on local, national and international levels with different degrees of coordination. ${ }^{1413}$ The recent Delphi study from the EPDWG confirmed marked heterogeneity of COVID-19 countermeasure implementation as of 20 March 2020, across 13 paediatric nephrology centres in 11 European countries, ${ }^{5}$ with case loads ranging from 4 to 680 infected patients per million (median 161 per million). This variability led us to hypothesise that growing pressures from increasing country-specific case loads were the main drivers for countermeasure implementation in our centres, and that differing numbers of infected patients might explain the heterogeneity in response rates among centres, consistent with general international trends amidst the COVID-19 pandemic. $^{514}$

However, the present study's comparisons of centre case loads with mean centre responses or with mean response rates of individual measures found no close correlation. Thus, pandemic phase alone cannot explain the observed heterogeneity of COVID-19 countermeasure implementation rates across European centres. We therefore treated countermeasure implementation as a complex process with multiple influencers. ${ }^{6}{ }^{15}$ In the conceptual framework of COM-B, countermeasure implementation rates likely represent the 'capability' (as 'regulation' and/ or 'guideline' policies) of their drivers (experts and/or hospital authorities) to allocate resources by opinion or rules, balancing pressure of the pandemic phase ('motivation' as 'communication' policies) and availability of resources ('opportunity' as 'environmental/social' policies).

Complex interactions between these factors in the BCW (figure 1) might better explain observed heterogeneities of implementation rates among different centres and measures. In this context, increased pressure from pandemic progression shifted expert opinion-based 
A)

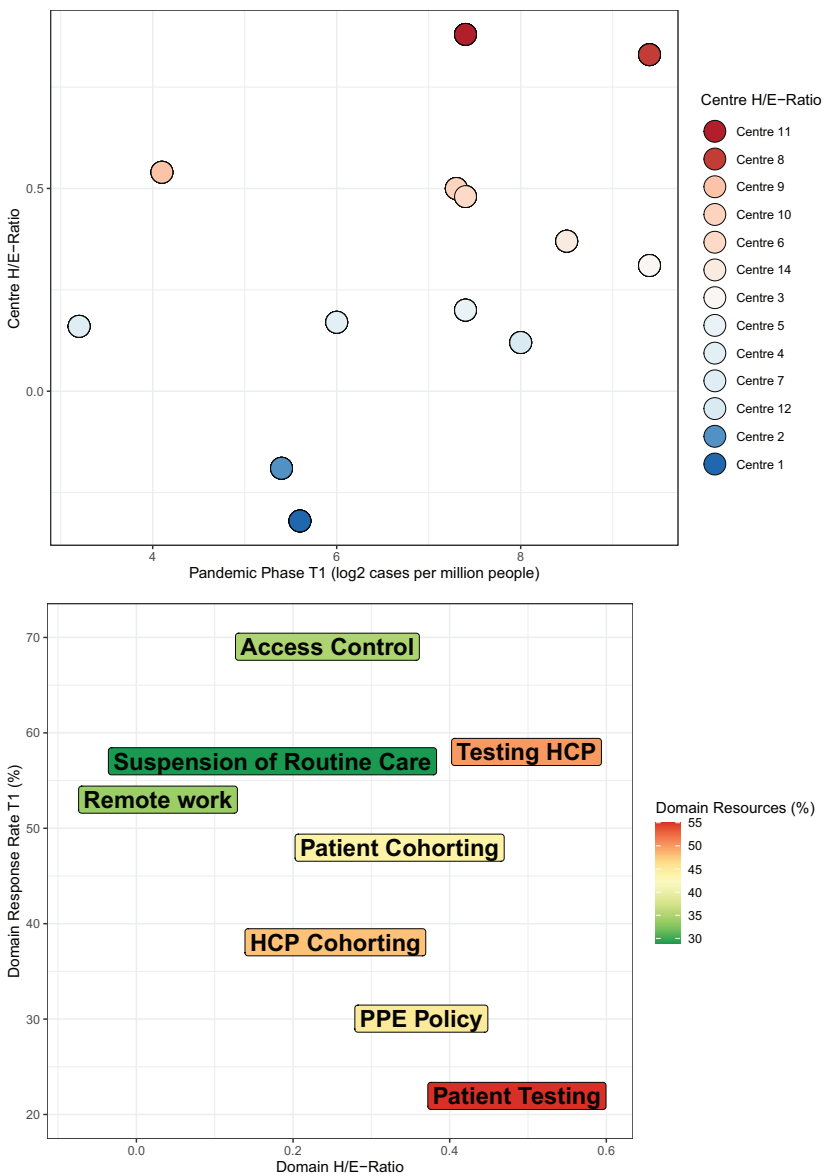

Figure 3 (A) Centre-specific ratios of hospital rules (red) to expert opinion (blue) $(\mathrm{H} / \mathrm{E}$ ratio) driving countermeasure implementation for each of the 13 European Pediatric Dialysis Working Group (EPDWG) centres as a function of infected patient case load (log2 of infected cases per million people), a measure of pandemic phase on 20 March 2020 (T1). (B) Domain response rates (implemented countermeasures per domain as \% of total) as a function of countermeasure resource dependency (mean of all estimates from each centre for each individual domain) for each of the eight countermeasure domains and their drivers (red, hospital rules; green, expert opinion) on 20 March 2020 (T1). Higher implementation rates of countermeasures with comparable resource dependency often correlated with higher hospital/ expert driver ratio (compare 'Testing HCP' to 'Patient Testing ' as opposed to comparison of 'Suspension of Routine Care' to 'Remote Work'). HCP, healthcare personnel; PPE, personal protective equipment.

decisions towards more formal hospital rules, likely to overcome growing barriers to additional countermeasure implementation that in part reflected increasing awareness of growth in resource dependency. This might further reflect transfer of decisions from a personal individual level to systemic levels with increasing moral injury and mental health issues due to constrictions in provision of care caused by inadequate resources. ${ }^{16}$ From the perspective of children requiring long-term kidney replacement therapy (dialysis or kidney transplantation), examples of resource dependency include increased
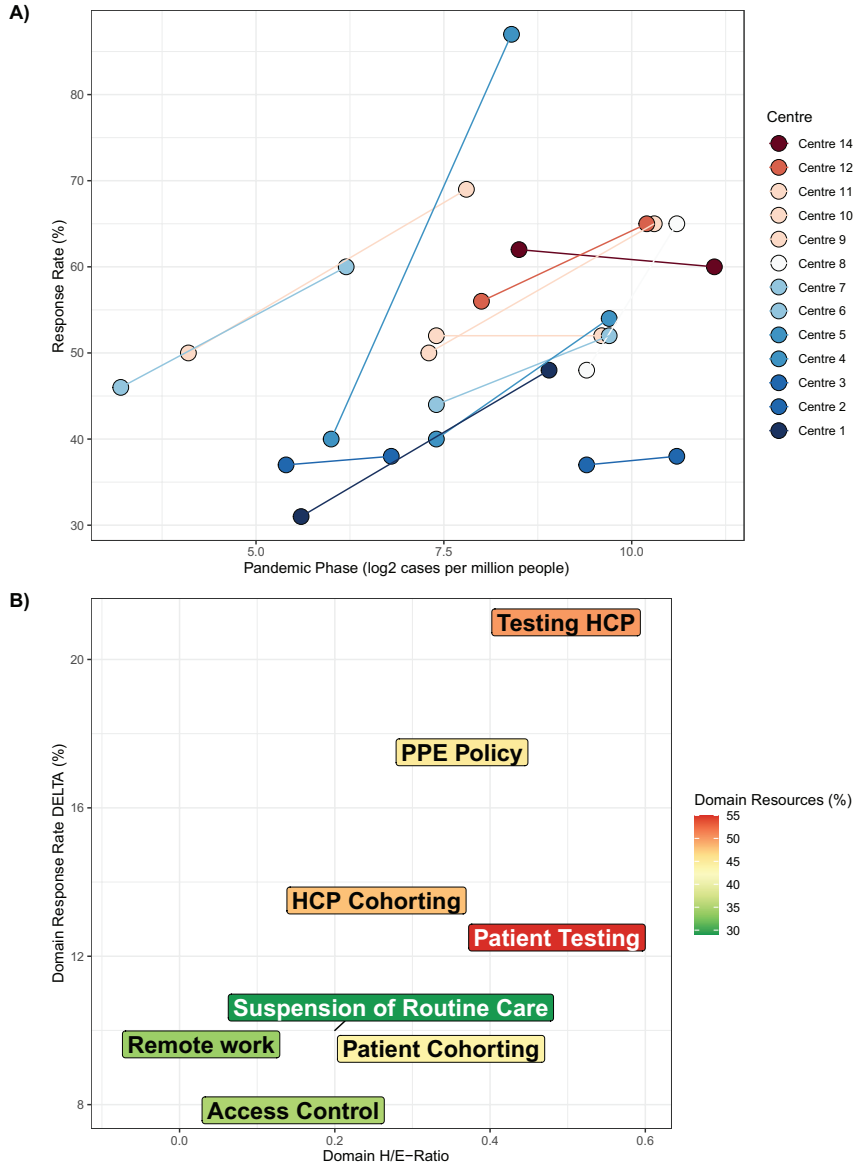

Figure 4 (A) Response rate dynamics were plotted for each centre versus dynamics of pandemic phase (log2transformed cases per million people) during the period of 20 March to 3 April 2020 (DELTA = difference of response rates/log2-transformed cases per million people between the two points in time). (B) Changes in response rates during the period of 20 March to 3 April 2020 (DELTA = difference of response rates/domain $\mathrm{H} / \mathrm{E}-$ Ratios between the two points in time) for each countermeasure domain were plotted against the average domain-specific hospital to expert ratio. Drivers for implementation are quantitated as relative domain resource dependency from low (green) to high (red). HCP, healthcare personnel; H/E, hospital to expert ratio; PPE, personal protective equipment.

difficulties in accessing medical care and travelling to hospitals for regular kidney function tests, drug concentration monitoring at specialised clinics and acquisition of medical supplies such as peritoneal dialysis fluids and equipment. ${ }^{17}$ In a February 2020 Chinese survey of caretakers of children requiring long-term kidney replacement therapy in the midst of the pandemic, these resource-dependent factors were thought by nearly $80 \%$ of participants to negatively affect their children's treatment, with one-third perceiving the effect as severe or extremely severe. ${ }^{17}$ EPDWG centre implementation rates of COVID-19 countermeasures, when regarded as changes of 'institutional behavior', thus reflected the ability of drivers at each centre to overcome local resource dependency. These changes, motivated by local 
perception of growing global medical need, led to diverse local rules and heterogeneous responses. Longitudinal assessment of countermeasure implementation from 20 March to 3 April supported the hypothesis that pressure from growing country-specific case loads increased local implementation of hospital rules, overcoming the initially inhibitory effects of locally perceived resource dependency of these measures, particularly measures with lower initial response rates. Initial inhibitory effects of locally perceived resource dependency might have diminished with the passage of time as medical supply deliveries and medical resource mobilisation have accelerated. These supplies are essential to carry out measures for pandemic control, protection of healthcare workers and mitigation of the severity of patient outcomes. ${ }^{18}$ Ordinarily, a shared body of scientific evidence ('what is right') underlies consensus procedures to harmonise institutional behavior' in response to medical challenges. Evidencebased medicine uses the best available evidence to help provide an optimal basis for decision-making according to individual circumstances and values. ${ }^{19}$ However, whereas COVID-19 countermeasure implementation rates increased at almost all EPDWG centres, overall response patterns among centres with similar case loads or at similar pandemic phase did not converge. Despite the pressure of pandemic progression, individual within-centre drivers appeared influenced by different perceptions of this pressure and by different local resource dependencies (and/ or awareness of those dependencies). This suggests other centre-specific and/or country-specific factors, beyond pandemic phase progression, that significantly influence countermeasure implementation. The COM-B and BCW models also allow systematic analysis of drivers for different behaviours and interventions on all levels, from individuals to national governments and civil societies.

Our analysis identified the non-medical influencers, media attitude and governmental strictness as important determinants of EPDWG centre responses to COVID-19 which might foster effective implementation of other medically relevant measures. ${ }^{20}$ Governmental interventions, in particular those aimed at social distancing, were recognised early in China as the most effective non-medical tool to 'flatten the curve' of the pandemic in several observations. ${ }^{1} 1321$ Similar interventions, ranging from banning large events to strict curfews, were implemented to varying degrees in European countries during the week of 20 March. Our study quantified these interventions and found that higher 'Governmental Strictness' correlated with increased centre responses, associated with reduced perception of resource dependency ('resource/cost awareness') of countermeasure implementation. Media dissemination of information can be incorporated in the COM-B and BCW models as a motivational driver for behaviour and decision-making on all social levels. ${ }^{20}$ This pertains especially to European countries attempting to contain the pandemic to the degree achieved in China, but in settings where governmental strictness effects on social distancing depend more on individual decisions and actions. ${ }^{1}$ Furthermore, important obstacles opposing a comprehensive European response to COVID-19 are being exposed, despite high level of political commitment. ${ }^{22}$ During the week of 20 March, the three widest circulation newspapers in each participating European country covered COVID-19 with $>75 \%$ of front page text. Centres in countries with more positive media attitude towards governmental strictness (based on front page articles) also demonstrated higher response rates, associated with higher perception of importance of expert opinion as driver for countermeasure implementation. This is in line with a Chinese study exploring new and traditional media use amidst the beginnings of the COVID-19 outbreak. ${ }^{23}$ Chao et al found that new media use with heavier engagement was associated with negative psychological outcomes, whereas viewings of heroic acts, speeches from experts and knowledge of the disease and prevention were associated with positive psychological impact. They conclude that timely public health communication from official sources might be beneficial in terms of general psychological health. ${ }^{23}$ The rapidly evolving shared knowledge base and emerging 'best practices' for counteracting COVID-19 in the European context allowed our study on EPDWG centre practice patterns, using COM-B and BCW models to describe behavioural drivers, to serve as a case study of institutional 'behavioral changes' under high pressure with insufficient available information. Under such conditions, we might expect that skills (but not knowledge) and tactics (but not strategy) will guide an individual's decisions and (measurable) actions. The same held true at the institutional level where, for example, varied initial policies on PPE and testing material led to nationwide export bans, prioritising local demand and production. ${ }^{3}$ Furthermore, differences in testing strategy inherent to differences in commercially available laboratory tests, especially those failing to detect low-level immune responses to SARS-CoV-2 in asymptomatic or mildly affected subjects, as well as those indicating false-positive results, ${ }^{24}$ might complicate decision guidance through other factors. Such mechanisms and interdependencies detected by our targeted statistical approach might increase understanding of still heterogeneous response patterns among countries with similar infection rates. This is in line with most countries having responded to this acute crisis with different tactics, often borrowed from non-medical sectors, in order to reduce transmission, increase local resources and contain medical, economical and other public threats accompanying this pandemic-whether being successful or not. ${ }^{25} 26$

As COVID-19 countermeasure implementation in the European context was not based on 'hard' scientific evidence, none of the implemented local policies can be objectively judged from a medical viewpoint as 'right' or 'wrong'. At time of submission 6 months after the initial Delphi exercise, there remains no strong evidence on efficacy of individual COVID-19 countermeasures pertaining to the European paediatric dialysis population. Recent 
Chinese consensus guidelines ${ }^{27}$ mentioned neither suspension of routine care nor testing strategies (for HCP and patients), although these measures were advocated as important to COVID-19 control. $^{28}$ The COVID-19 outbreak in a German paediatric dialysis centre ${ }^{29}$ also highlights the importance of adequate testing, tracing and monitoring strategies for successful outbreak containment and prevention in the hospital setting. However, in the mean time, at least one comprehensive systematic review has been published. This meta-analysis supporting physical distancing and face mask use provides the best evidence yet available, given the body of literature generally lacking robust randomised trials. ${ }^{30}$

This exploratory work provides a framework containing the most important domains that emerged during the lockdown phase in paediatric dialysis centres across Europe. Put into general context, these domains may provide some of the most important guiding principles but lack general completeness and might be rapidly outdated. However, put into perspective of the BCW and the COM-B model, these domain sets present an important framework for regular and multilayered reassessment by policymakers and clinicians to provide a basis for further decision-making and evolving awareness of possible limitations and subliminal influential factors.

As the results of this work reflect, the issues encountered in the course of providing the best possible care for our patients during a pandemic are multilayered and dependent on internal and external factors that vary across different cultural, legislative, economical and geographical areas. Moreover, these influences are likely to be subject to changing directives of changing degrees of influence over time. Standardised responses as usually provided by policymakers and societal guidelines do not consider these manifold factors and their dynamics in order to provide the best possible evidence-based medical care during a pandemic. ${ }^{31}$

In such deleterious scenarios where single patient groups and countries and continents are affected, the current gold standards for guidelines and policies as proposed by evidence-based medicine might not be applicable, and even cause negative effects on specific subgroups. Carefully graded stages considering legislative, economical and cultural differences need to set the framework for guiding patient care in accordance with increasing knowledge of an emerging evidence base. Policymakers and healthcare providers must maintain awareness of newly emerging influence factors, especially if readily fit into the subcategories communication, legislation, environmental/social, while sustaining flexibility to respond to the capability, opportunity and/ or motivation for change. These graded stages should be selected in accordance with current events, individually applied in different geographical, economical and cultural subspaces and continuously re-evaluated with progression of time and events. Therefore, continuous and regular multilayered reassessment of the most meaningful domains is necessary. ${ }^{31}$
The major strength of this study lies in its being the first to evaluate the most important drivers of behaviours conducive to counteracting the COVID-19 pandemic during the first week of public curfews. During this time, we applied an accepted model of behavioural change (the COM-B model and BCW) to explore a unique snapshot of 14 paediatric dialysis centres in 12 European countries with case loads ranging from 4 to 680 infected patients per million. The strength of our novel study approach may also inherently limit the interpretation of our results due to the absence of comparable studies with which to compare. The interpretation of this study's results is further limited by a small number of participating centres representative of paediatric dialysis, but perhaps not equally representative of other medical responses to the COVID-19 pandemic. In addition, the number of participants per centre was limited to one clinician only in order to facilitate rapid communication and data acquisition. However, given the small number of participants and exploratory mixed methods character of this study, statistical tests and their corresponding $p$ values should be interpreted with caution. Moreover, local case loads of the surveyed centres may not reflect overall disease burdens of the respective countries and/or other medical specialties, with higher numbers of infections and/or patients at risk.

Countermeasures evaluated in this study most likely reflect similar countermeasures in other medical specialties, as current mitigating approaches to COVID-19 all rely on the same measures, such as physical distancing, PPE and testing capacities.

This study may also serve as a basic framework for research and awareness of factors influencing exit strategies for the implemented countermeasures, providing clinicians and policymakers with guidelines for early and structured adaptation to changing or fluctuating conditions. Ruktanonchai $e t a l^{32}$ underline the importance of such guidelines in their modelling study which shows that relaxation of countermeasures by one country before others do so could lead to disease resurgence across Europe about 5 weeks earlier than otherwise. Their study also highlights the importance of key countries, such as France, Germany, Italy and Poland, in continental resurgence of disease due to heterogeneous approaches to mobility restriction. ${ }^{32}$

Nevertheless, heterogeneity of countermeasure implementation can be expected to continue among European centres until ongoing 'catch-up implementation' saturates response rates, as limited by local availability and resources. Although our study provides no solutions to that problem, our 'mechanistic' work does provide a mirror for the weak evidence basis underlying current practice patterns. ${ }^{1}$ Understanding limitations of current approaches to selection and implementation of COVID-19 countermeasures might help reassess those practices with open minds, allowing rapid 'institutional behavior changes' in response to emerging evidence on efficacy from controlled clinical trials. These will also 
provide evidence-based knowledge to optimise nonmedical interventions during the COVID-19 pandemic.

\section{Author affiliations}

${ }^{1}$ Division of Pediatric Nephrology and Gastroenterology, Department of Pediatrics and Adolescent Medicine, Comprehensive Center for Pediatrics, Medical University of Vienna, Vienna, Austria

${ }^{2}$ Ludwig Boltzmann Institute Digital Health and Patient Safety, Medical University of Vienna, Wien, Austria

${ }^{3}$ Section for Outcomes Research, Center for Medical Statistics, Informatics, and Intelligent Systems, Medical University of Vienna, Vienna, Austria

${ }^{4}$ Ludwig Boltzmann Institute for Arthritis and Rehabilitation, Vienna, Austria

${ }^{5}$ Research Platform Data Science, University of Vienna, Vienna, Austria

${ }^{6}$ Pediatric Nephrology, Center for Child and Adolescent Medicine, Heidelberg University, Heidelberg, Germany

${ }^{7}$ Department of Pediatric Nephrology, University Hospital Vall d'Hebron, Barcelona, Spain

${ }^{8}$ Division of Pediatric Nephrology, Gazi University Faculty of Medicine, Ankara,

Turkey

${ }^{9}$ Pediatric Center, Institute of Clinical Medicine, Vilnius University, Vilnius, Lithuania

${ }^{10}$ Department of Pediatric Nephrology, KfH Children's Kidney Center, Marburg,

Germany

${ }^{11}$ Pediatric Nephrology, Dialysis and Transplant Unit, La Fondazione IRCCS Ca

Granda Ospedale Maggiore di Milano Policlinico, Milano, Italy

${ }^{12}$ Department of Pediatric Nephrology, Hôpital Femme Mère Enfant, Hospices Civils de Lyon, Lyon, France

${ }^{13}$ Renal Unit, UCL Great Ormond Street Hospital for Children NHS Foundation Trust and Institute of Child Health, London, UK

${ }^{14}$ Department of Pediatric Nephrology, Mitera Children's Hospital, Athens, Greece

${ }^{15}$ Department of Pediatric Nephrology, Utoped, Universitair Ziekenhuis Gent, Ghent, Belgium

${ }^{16}$ Dialysis Unit, Department of Pediatrics, IRCCS Giannina Gaslini, Genoa, Italy

${ }^{17}$ Department of Pediatric Nephrology, University Hospital Motol, Prague, Czech Republic

${ }^{18}$ Department of Pediatric Nephrology, Medical University of Gdansk, Gdansk, Poland

${ }^{19}$ Division of Nephrology and Vascular Biology Research Center, Beth Israel

Deaconess Medical Center, Department of Medicine, Harvard Medical School,

Boston, Massachusetts, USA

Contributors FE and CA conceptualised and designed the study, had full access to all study data and take responsibility for data integrity, accuracy and analysis. They drafted the initial manuscript, analysed and interpreted the data and reviewed and revised the manuscript. Data collection and critical manuscript revision for important intellectual content were conducted by SLA, CPS, VR, TS, AC, GA, AJ, GK, $\mathrm{FP}, \mathrm{AE}, \mathrm{BR}, \mathrm{RS}, \mathrm{CJS}, \mathrm{JV}, \mathrm{EV}, \mathrm{KV}, \mathrm{AZ}$ and SB. All authors approved the final manuscript as submitted.

Funding The authors have not declared a specific grant for this research from any funding agency in the public, commercial or not-for-profit sectors.

Competing interests None declared.

Patient consent for publication Not required.

Provenance and peer review Not commissioned; externally peer reviewed.

Data availability statement All data relevant to the study are included in the article or uploaded as supplementary information. No additional data available.

Supplemental material This content has been supplied by the author(s). It has not been vetted by BMJ Publishing Group Limited (BMJ) and may not have been peer-reviewed. Any opinions or recommendations discussed are solely those of the author(s) and are not endorsed by BMJ. BMJ disclaims all liability and responsibility arising from any reliance placed on the content. Where the content includes any translated material, BMJ does not warrant the accuracy and reliability of the translations (including but not limited to local regulations, clinical guidelines, terminology, drug names and drug dosages), and is not responsible for any error and/or omissions arising from translation and adaptation or otherwise.

Open access This is an open access article distributed in accordance with the Creative Commons Attribution Non Commercial (CC BY-NC 4.0) license, which permits others to distribute, remix, adapt, build upon this work non-commercially, and license their derivative works on different terms, provided the original work is properly cited, appropriate credit is given, any changes made indicated, and the use is non-commercial. See: http://creativecommons.org/licenses/by-nc/4.0/.

ORCID iDs

Fabian Eibensteiner http://orcid.org/0000-0001-5012-3082

Tanja Stamm http://orcid.org/0000-0003-3073-7284

\section{REFERENCES}

1 Anderson RM, Heesterbeek $\mathrm{H}$, Klinkenberg D, et al. How will countrybased mitigation measures influence the course of the COVID-19 epidemic? Lancet 2020;395:931-4.

2 Chen S, Yang J, Yang W, et al. COVID-19 control in China during mass population movements at new year. Lancet 2020;395:764-6.

3 Feng S, Shen C, Xia N, et al. Rational use of face masks in the COVID-19 pandemic. Lancet Respir Med 2020;8:434-6.

4 Flahault A. COVID-19 cacophony: is there any orchestra conductor? Lancet 2020;395:1037.

5 Eibensteiner F, Ritschl V, Ariceta G, et al. Rapid response in the COVID-19 pandemic: a Delphi study from the European pediatric dialysis Working group. Pediatr Nephrol 2020;35:1669-78.

6 Michie S, van Stralen MM, West R. The behaviour change wheel: a new method for characterising and designing behaviour change interventions. Implement Sci 2011;6:42.

7 Ritschl V, Lackner A, Boström C, et al. I do not want to suppress the natural process of inflammation: new insights on factors associated with non-adherence in rheumatoid arthritis. Arthritis Res Ther 2018;20:234.

8 Johnson RB, Onwuegbuzie AJ, Turner LA. Toward a definition of mixed methods research. J Mix Methods Res 2007;1:112-33.

9 Johnson RB, Onwuegbuzie AJ. Mixed methods research: a research paradigm whose time has come. Educational Researcher 2004;33:14-26.

10 Creswell JW. Research design: qualitative, quantitative, and mixed methods approaches. SAGE Publications, 2013.

11 European centre for disease prevention and control (ECDC) COVID-19 situation update worldwide, as of 6 April 2020. Available: https://www.ecdc.europa.eu/en/geographical-distribution-2019ncov-cases [Accessed 6 Apr 2020].

12 Eurostat, Europe's population on January the 1st 2019. Available: https://ec.europa.eu/eurostat/tgm/table.do?tab=table\&plugin=1\& language $=$ en \&pcode=tps00001 [Accessed 6 Apr 2020]

13 Pan A, Liu L, Wang C, et al. Association of public health interventions with the epidemiology of the COVID-19 outbreak in Wuhan, China. JAMA 2020;323:1915-23.

14 Koh D, Goh HP. Occupational health responses to COVID-19: what lessons can we learn from SARS? J Occup Health 2020;62:e12128.

15 Essack SY, Sartorius B. Global antibiotic resistance: of contagion, confounders, and the COM-B model. Lancet Planet Health 2018;2:e376-7

16 Greenberg N, Docherty M, Gnanapragasam S, et al. Managing mental health challenges faced by healthcare workers during covid-19 pandemic. BMJ 2020;368:m1211

17 Zhao R, Zhou Q, Wang X-W, et al. COVID-19 outbreak and management approach for families with children on long-term kidney replacement therapy. Clin J Am Soc Nephrol 2020;15:1259-66.

$18 \mathrm{Ji}$ Y, Ma Z, Peppelenbosch MP, et al. Potential association between COVID-19 mortality and health-care resource availability. Lancet Glob Health 2020;8:e480.

19 Djulbegovic B, Guyatt G. Evidence vs consensus in clinical practice guidelines. JAMA 2019:725. doi:10.1001/jama.2019.9751

20 Garrett L. COVID-19: the medium is the message. Lancet 2020;395:942-3.

21 Cowling BJ, Ali ST, Ng TWY, et al. Impact assessment of nonpharmaceutical interventions against coronavirus disease 2019 and influenza in Hong Kong: an observational study. Lancet Public Health 2020;5:e279-88

22 Anderson M, Mckee M, Mossialos E. Covid-19 exposes weaknesses in European response to outbreaks. BMJ 2020;368:m1075.

23 Chao M, Xue D, Liu T, et al. Media use and acute psychological outcomes during COVID-19 outbreak in China. J Anxiety Disord 2020;74:102248.

24 Reiter T, Pajenda S, Wagner L. Covid-19 serology in nephrology health care workers. medRxiv2020;20136218.

25 Cramton P, Ockenfels A, Roth AE, et al. Borrow crisis tactics to get COVID-19 supplies to where they are needed. Nature 2020;582:334-6.

26 Okazawa M, Suzuki S. Japanese tactics for suppressing COVID-19 spread. Public Health 2020;186:6-7. 
27 Shen Q, Wang M, Che R, et al. Consensus recommendations for the care of children receiving chronic dialysis in association with the COVID-19 epidemic. Pediatr Nephrol 2020;35:1351-7.

28 Schneider EC. Failing the Test - The Tragic Data Gap Undermining the U.S. Pandemic Response. N Engl J Med 2020;383:299-302.

29 Schwierzeck V, König JC, Kühn J. First reported nosocomial outbreak of severe acute respiratory syndrome coronavirus 2 (SARS-CoV-2) in a pediatric dialysis unit. Clin Infect Dis 2020 doi:10.1093/cid/ciaa491

30 Chu DK, Akl EA, Duda S, et al. Physical distancing, face masks, and eye protection to prevent person-to-person transmission of
SARS-CoV-2 and COVID-19: a systematic review and meta-analysis. Lancet 2020;395:1973-87.

31 Mak TK, Lim JC, Thanaphollert P, et al. Global regulatory agility during covid-19 and other health emergencies. BMJ 2020;369:m1575.

32 Ruktanonchai NW, Floyd JR, Lai S, et al. Assessing the impact of coordinated COVID-19 exit strategies across Europe. Science 2020;369:1465-70. 